\title{
Psychiatric hospital reform in low- and middle-income countries: a systematic review of literature
}

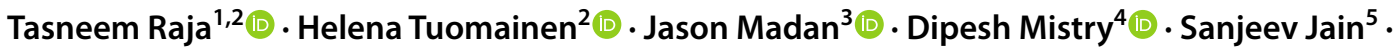 \\ Kamala Easwaran ${ }^{2,6}$ (D) Swaran P. Singh ${ }^{7}$ (D)
}

Received: 6 December 2020 / Accepted: 7 April 2021 / Published online: 21 April 2021

(c) The Author(s) 2021

\begin{abstract}
Purpose Psychiatric hospitals or mental asylums grew across the world in the colonial era. Despite concerns over quality of care and human rights violations, these hospitals continue to provide the majority of mental health care in most low- and middle-income countries (LMICs). We sought to review the evidence of reform of mental hospitals and associated patient outcomes.

Methods We adopted an integrative review methodology by including experimental and non-experimental research. The review protocol was registered on PROSPERO (CRD42019130399). A range of databases and systematic hand searches were conducted by two independent reviewers. Research conducted between 1980 and May 2019, that focused on any aspect of reform in mental hospitals for adults (age 18 and upwards) with severe mental illness and published in English, were considered.

Results 16 studies were included in the review. 12 studies met inclusion criteria, and four additional reports emerged from the hand search. Studies covered-India, China, South Africa, Grenada, Georgia, Sri Lanka, Argentina and Brazil. Key findings emphasise the role of judicial intervention as a critical trigger of reform. Structural reform composed of optimisation of resources and renovations of colonial structures to cater to diverse patient needs. Process reforms include changes in medical management, admission processes and a move from closed to open wards. Staff engagement and capacity building have also been used as a modality of reform in mental hospital settings.

Conclusion There is some documentation of reform in psychiatric hospitals. However, poor methodological quality and variation in approach and outcomes measured, make it challenging to extrapolate specific findings on process or outcomes of reform. Despite being integral service providers, psychiatric hospitals still do not adopt patient centric, recovery-oriented processes. Hence, there is an urgent need to generate robust evidence on psychiatric reform and its effect on patient outcomes.
\end{abstract}

Keywords Psychiatric hospitals · Low- and middle-income countries · Transforming psychiatric hospitals $\cdot$ Reform of mental hospitals

Tasneem Raja

tasneemraja@gmail.com; traja@ tatatrusts.org;

T.Raja@warwick.ac.uk

Helena Tuomainen

helena.tuomainen@warwick.ac.uk

Sanjeev Jain

sjain.nimhans@nic.in; sjain.nimhans@gmail.com

Kamala Easwaran

kamala@sumunum.com

1 Tata Trusts, World Trade Center, Cuffe Parade, Mumbai 400005, India

2 Mental Health and Wellbeing, Warwick Medical School, University of Warwick, Coventry, England
3 Centre for Health Economics, Warwick Medical School, University of Warwick, Coventry, England

4 Warwick Clinical Trials Unit, University of Warwick, Coventry, England

5 Molecular Genetics Laboratory, Department of Psychiatry, National Institute of Mental Health and Neurosciences, Hosur Road, Bangalore 560029, India

6 Founder Sumunum Foundation, Chennai, India

7 Centre for Mental Health and Wellbeing Research, University of Warwick, Coventry, England 


\section{Introduction}

Mental asylums are a hallmark of the globalization of psychiatry. Established globally during the colonial period, they continue to provide care for the severely mentally ill. They account for a majority of mental health care available in Low- and Middle-Income Countries (LMICs). Mental hospitals consume a bulk of the financial resources allocated for mental health. Despite the high admission rates into psychiatric care settings $(99.1$ per 100,000), the number of hospital beds per 100,000 is only 11.3 , with over $18 \%$ of bed capacity being occupied by individuals with long-term care needs. With only nine mental health professionals per 100,000 people, the burden of caregiving is high [1]. Care provided in mental hospitals is shaped by a range of factors including legal reforms of the late twentieth and early twenty-first century [1-3].

Psychiatric hospitals remained an area of interest to the scientific community for a large part of the twentieth century; however, the interest sharply declined over the last three decades $[4,5]$. Further, the emerging field of global mental health prioritized research on community mental health [6] particularly on common mental disorders and depression while neglecting mental hospitals. Hospitals and long-stay institutions do not feature in the top 25 Grand Challenges in Global Mental Health $[7,8]$. The fight to improve the appalling conditions and reduce incidence of abuse in these hospitals has been left to media, non-governmental organizations and human rights commissions [9-13]. There is a disconnect between this community-based focus of global mental health and the plight of the severely mentally ill who are often behind closed walls of institutions. Mental hospitals continue to remain an important provider of care and there is an urgent need to reform practices to improve quality of care and reclaim dignity for service users. LMICs need pragmatic and evidence-based approaches to psychiatric hospital reform, where there is a balance and optimisation of resources spent on community care vis-a-vis expenditure on psychiatric institutions [2].

\section{Objective}

Given this backdrop, a systematic review of literature synthesizing research on psychiatric hospital reform, particularly in LMICs is essential to frame stronger, more appropriate reform programmes. The review aimed to understand the process and outcome of psychiatric hospital reform in LMICs by:
- Distilling evidence and scientific literature around mental hospital reform in LMICs and documenting process and outcome of reform.

- Understanding the impact of structural and process reform of psychiatric hospitals on patient outcomes in LMICs.

- Identifying gaps in current evidence and research with regard to the reform of psychiatric institutions in LMIC country settings.

\section{Methods}

We adopted an integrative review methodology for this study. It includes the four steps of a systematic process i.e. search, appraisal, synthesis and analysis. It allows for inclusion of both experimental and non-experimental research. The review protocol was registered on PROSPERO CRD42019130399. We followed the Preferred Reporting Items for Systematic Reviews and Meta-Analyses (PRISMA) Statement and Consolidated Standards of Reporting Trials (CONSORT) Statement [14]. This review was undertaken as part of the Structured Individualised inTervention and Recovery (SITAR) study, which is embedded in a larger programme on psychiatric hospital reform called Udaan. Udaan is a partnership of Tata Trusts with government of Maharashtra, formalised through a Memorandum of Understanding, to develop the Regional Mental Hospital Nagpur (RMHN) as a centre of excellence through systematic reform of the hospital. Udaan (which in Hindi mean to 'soar') comprises four key reform elements: structural (refurbishing old colonial infrastructure to meet current service user needs), process (standardising clinical and non-clinical processes of the hospital), capacity building (standard training for different levels of hospital staff) and introduction of individual need-based, recovery-oriented, service package for patients [15]. Udaan defines psychiatric hospital reform as a care transformation process across the four domains of structural reform, process reform capacity building of staff and an individual patient services package. We have used this framework to operationally define reform for the purpose of this review. The SITAR study embedded within the Udaan program is a two-arm pragmatic randomised control trial which tests if an individual patient service package improves outcomes amongst long-stay in-patients in comparison to larger psychiatric hospital reform [15].

\section{Eligibility criteria}

The review sought to identify papers that studied mental hospitals or similar care facilities in the 137 LMICs. Research conducted between 1980 and May 2019, that focused on 
any aspect of reform for adults (age 18 and upwards) with severe mental illness and published in English were considered. In addition, we did a citation search of all included publications. Studies excluded were: non-English publications, studies from high income countries, interventions in general hospital and community settings for adolescents/ children (below age 18), and those that studied non-Severe Mental Disorders (SMDs). Studies published before 1980 were also excluded. The detailed PICOs for the review are in Table 1, below.

\section{Search strategy and data sources}

A two-pronged search strategy was used (a) database search and (b) hand searching to identify relevant studies.

We searched five databases: Medline, PsycINFO, Web of Science, Scopus and Cochrane using the key words and combinations reflected in Table 2 below. We used a country specific search since the combination of key words for LMICs does not appear readily on databases. We conducted the search between Nov 2019 and February 2020 for all the databases. For the hand search, we examined the reference lists of all identified studies.

\section{Data extraction and quality assessment}

The first author (TR) ran the primary search, assessed eligibility criteria for all retrieved papers and assessed the quality of all included studies. The first author also extracted data for all included studies with $100 \%$ of the sample being extracted independently by another author (KE). RATS (relevance, appropriateness, transparency and soundness) qualitative research review guidelines were used for the quality assessment of the seven qualitative studies. The RATS scale comprises 25 questions that assess the relevance of the research question, appropriateness of the methods used,
Table 1 PICOS for the systematic literature review

Table 2 Search strategy and syntax

\begin{tabular}{ll}
\hline Population & People living in an institute \\
& Mental hospitals/psychiatric hospitals/asylums/psychiatric institutions in Low- and \\
& Middle-Income Country (LMICs) as defined by the World Bank \\
Intervention & Intervention in the institutional setting \\
& Transition/reform/change/modernization/improvement/de-institutionalization \\
Comparator & People who have not received the intervention or to the setting prior to the intervention \\
& A comparator is not necessary \\
Outcomes & Change in patient level indicators-symptoms, functionality, disability, social interac- \\
& tion, quality of life (any relevent measure/scale). Process indicators, such as length of \\
& stay and number of admission episodes \\
Study design & Randomized and non-randomized study designs (all publications) \\
& From 1980 till date
\end{tabular}

\begin{tabular}{lc}
\hline Search terms & Resources \\
\hline Population & \\
Adults & \\
Setting & \\
Mental hospital & \\
Psychiatric hospital & Bibliographic \\
Mental asylums & and journal \\
& databases \\
Psychiatric institutions & APA PsychINFO \\
& PubMed/medline \\
Intervention & Cochrane reviews \\
Reform & Web of science \\
Change & Scopus \\
Modernisation & \\
Improvement & \\
Deinstitutionalisation & \\
Location & \\
Low and middle-income countries (lower, mid, and upper mid income) as defined by & \\
the World Bank-137 countries & \\
\hline
\end{tabular}


transparency of the study and methods and soundness of the approach used for interpretation of findings. For the purpose of this review, each question on the RATS scale was assigned a binary value (yes-1 point and no with 0 points) to effectively make a judgement on the quality of the included qualitative research papers. This approach was drawn from a previous systematic review using multiple types of studies $[16,17]$. The Effective Public Health Practice Project (EPHPP) [18] was used to assess the quality of the five quantitative studies included in the review. The four reports included from the citation search of included studies were not assessed for quality since they are reports, three of them are country reports and one is a programme report.

Data were extracted and tabulated independently by two authors (TR and KE) for all papers meeting the eligibility criteria. The data extraction tool was developed by the first author (TR) and was modeled on the data extraction templates for RCTs and non-RCTs [19]. The tool comprised the following categories: general information (title of the study, study authors, type of study, journal of publication, year of publication, country of study), intervention setting (type of facility, study period, number of patients in the study, length of stay along with the admission and discharge process), costing details (annual budget of the institution), reform components (triggers for reform, elements of reform and cost of reform), and outcomes (patient data on clinical, social and functional outcomes).

\section{Results}

802 studies were identified through the database search of which, following exclusion of duplicates, abstract reviews, and full-text reviews 12 studies met the inclusion criteria. The hand search yielded four additional reports (Fig. 1). Of the 16 studies included in this review, seven were varied qualitative studies including, two case studies, one personal reflection, one ethnographic study, one observational study and one historical study. Five of the 16 included articles and reports were quantitative studies. Of these, two were randomised controlled trials, one was a non-randomised control trial, one case-control study and one quasi-experimental study. The four publications found through citation search included three country-level reports and one programme report. Quality assessment indicated high variability with nine of the 12 assessed studies as weak, two as moderate and one as high quality.

\section{Data synthesis}

A preliminary synthesis was developed using tabulation. Data were directly reported and cumulated where possible for quantitative variables. For qualitative data, emergent themes were drawn out and a vote count was undertaken to identify the frequency with which themes appeared. The studies covered a publishing period from 1994 to 2017 and represented eight countries: India [19-23], China [24-26], South Africa [27, 28], Brazil [29, 30], Argentina [31], Grenada [32], Georgia [33] and Sri Lanka [34]. All of the studies were based in state-run psychiatric hospitals. A total of 112 hospitals were covered through these studies, out of which, there were 60 unique hospital settings (studies in India were done in the same hospital). The number of hospitals covered per country ranged from 47 in India to one each in Grenada and Sri Lanka, [19-24, 26-36]. A high variability was found in the number of patients/number of beds with some studies covering as low as 10 patients [27] and the highest being 237 from India [22] Three qualitative studies do not mention any numbers $[19,20,33]$. Since there is high variability in the numbers reported, no further analysis was undertaken Characteristics of all included studies are reported in Table 3 below.

The qualitative synthesis enabled a more nuanced understanding of reform processes and outcomes. The sections below elucidate key findings on triggers for reform, key elements of structural and process reform, staff enrichment and capacity building programmes, and outcomes of reform.

\section{Triggers for reform}

12 studies described a trigger for reform in mental hospital settings [19, 21, 23, 24, 27-32, 34, 36]. The reasons that triggered reform are listed in Table 4 and include poor quality of care particularly for long-stay patients in mental hospitals. India and Argentina report judicial intervention as a key trigger for reform in colonial era mental hospitals.

\section{Elements of reform}

Elements of reform described in the studies were categorised into structural reform (refurbishment of hospital infrastructure), process reform (reform of clinical and non-clinical hospital processes) and capacity building/ training of hospital staff. Seven $(43.75 \%)$ of the 16 studies included in the review reported structural elements of reform, captured in Table 5. In India, hospital infrastructure was improved [19, 21, 23], and community housing services established [20, 22]. In Georgia, a large 250-bedded hospital was closed and, in its place, multiple smaller 40-bedded units were established for long-stay patients [33]. In Argentina, hospital infrastructure was used to initiate half-way homes [31].

Process reform was reported in 14 (87.5\%) studies. This has been categorised and reported in Table 6. We have discussed these reforms by each country in this section to provide a comprehensive picture. 


\section{Study selection flow chart}

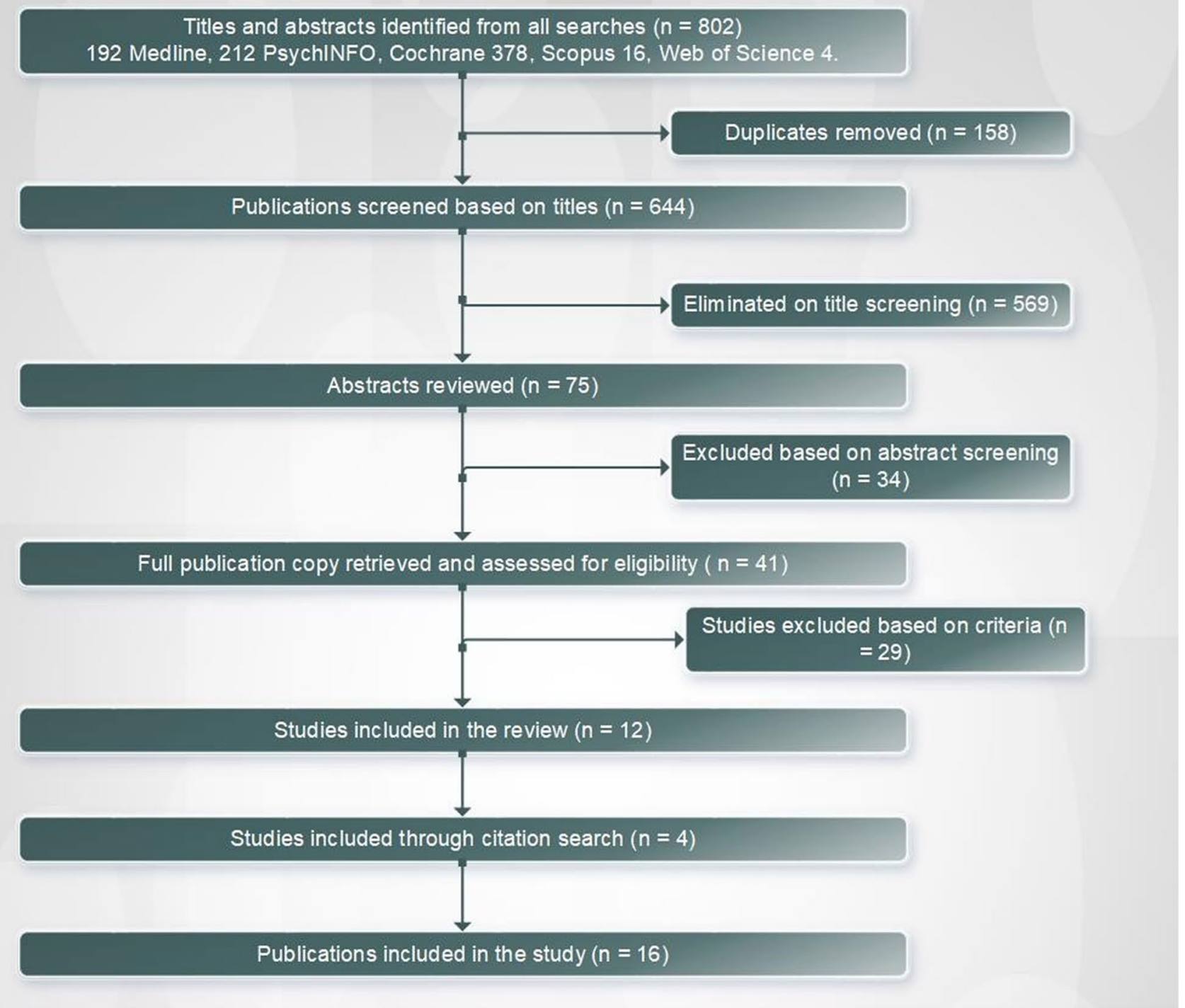

Fig. 1 Study selection flow chart

There were several process reforms initiated in Indian mental hospitals, including reforms in medical management [21], There was a shift in process of admissions with hospitals moving away from admissions through a legal intervention to voluntary admissions $[21,23]$. There was an initiation of more open wards as evidenced in two countrylevel reports, shift in restraint and seclusion processes, and a reduction in use off custodial cells for isolation [21, 23]. Simultaneously, community-based services linked to mental hospitals were also initiated [23]. An introduction of psychosocial interventions along with a focus on Activities of
Daily Living (ADL) and rehabilitation services including employment was reported [20, 22] along with greater inclusion and involvement of family members in the treatment and care process [21].

In China, process reforms in mental hospitals saw a move from closed to open wards [25], and initiation of communitybased services in tandem with mental hospitals [26]. Further, patient involvement in hospital management and promotion of non-hierarchical relationships between staff and patients through a structured engagement process was reported [24]. Psychosocial interventions were introduced [24-26] along 


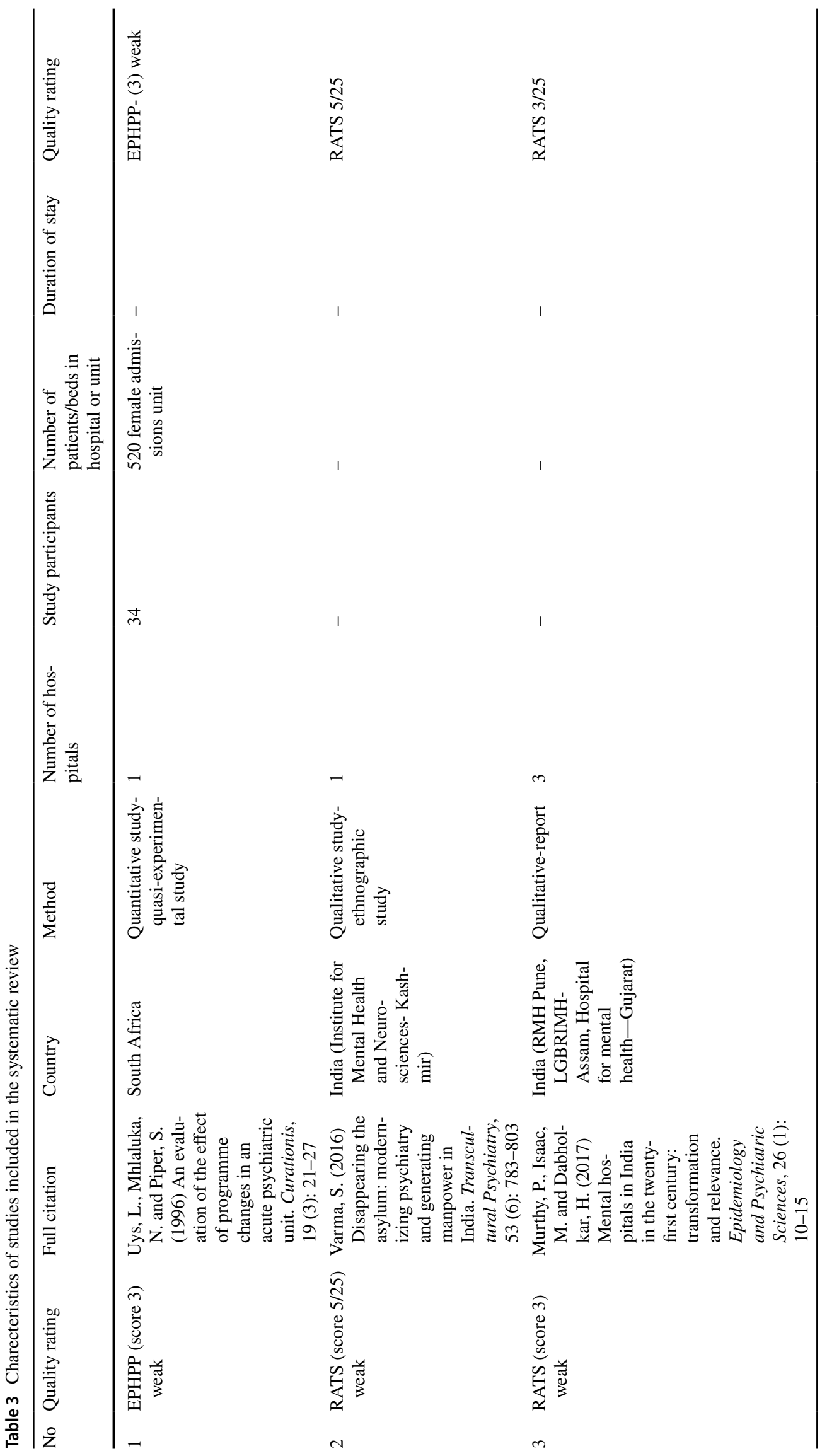




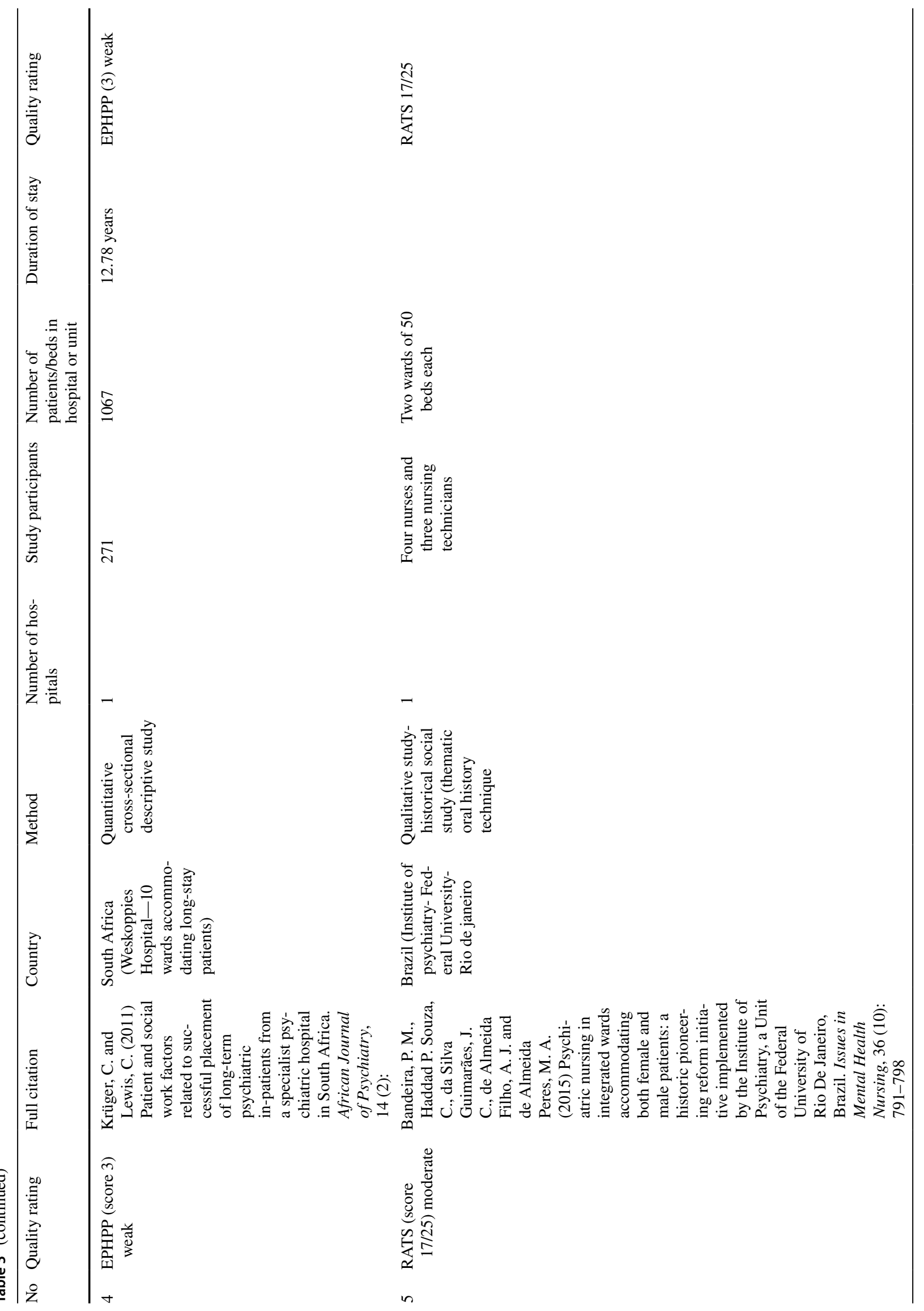




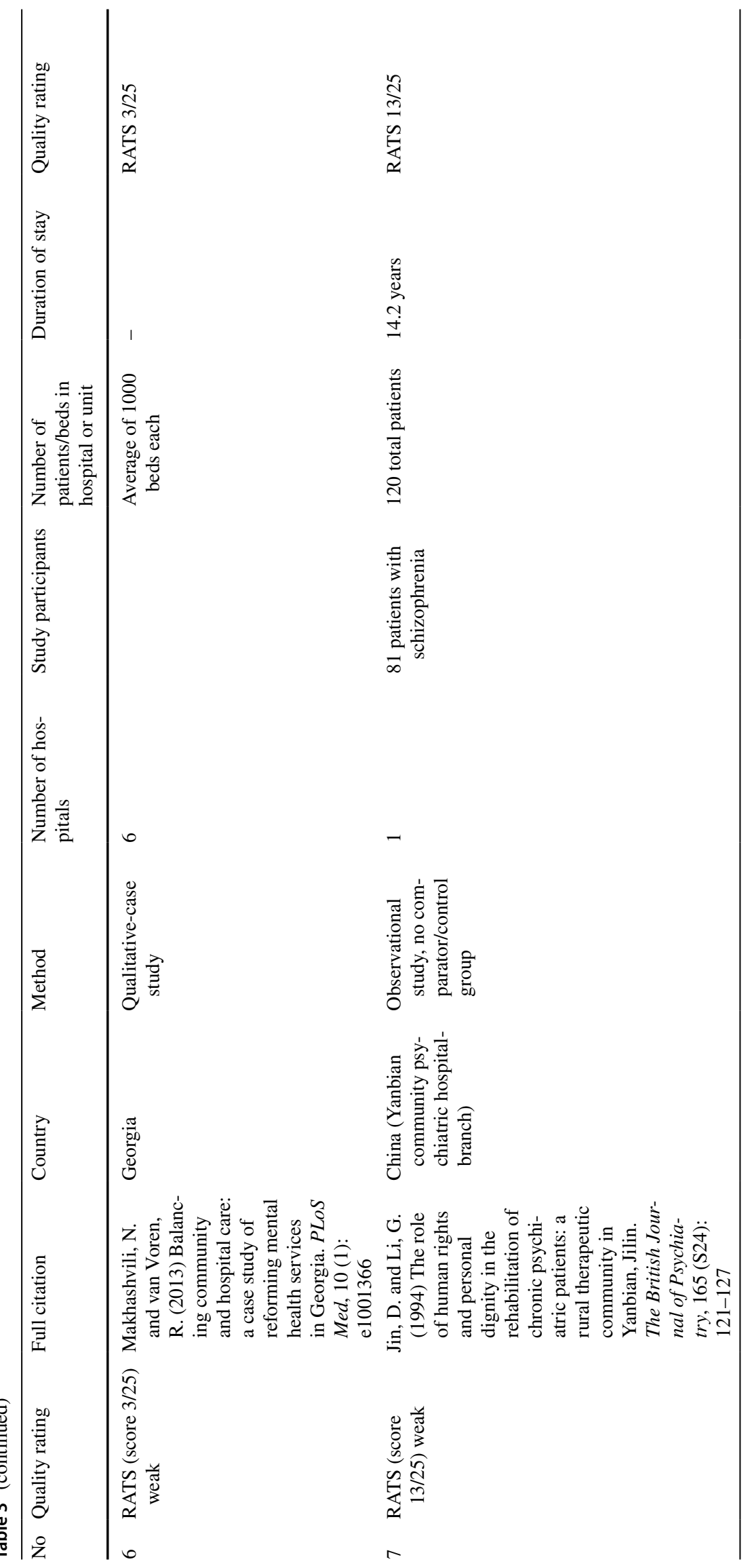




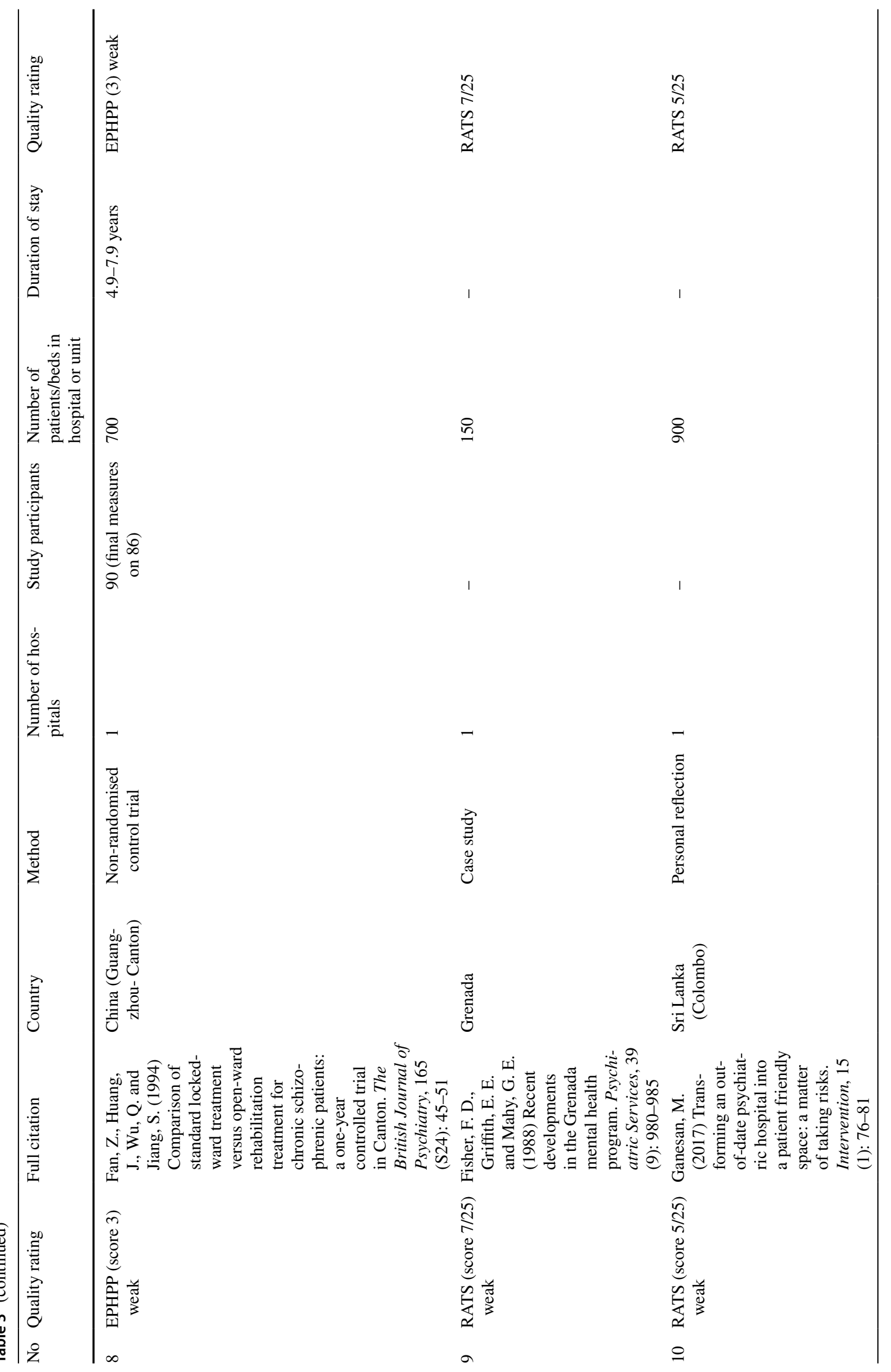




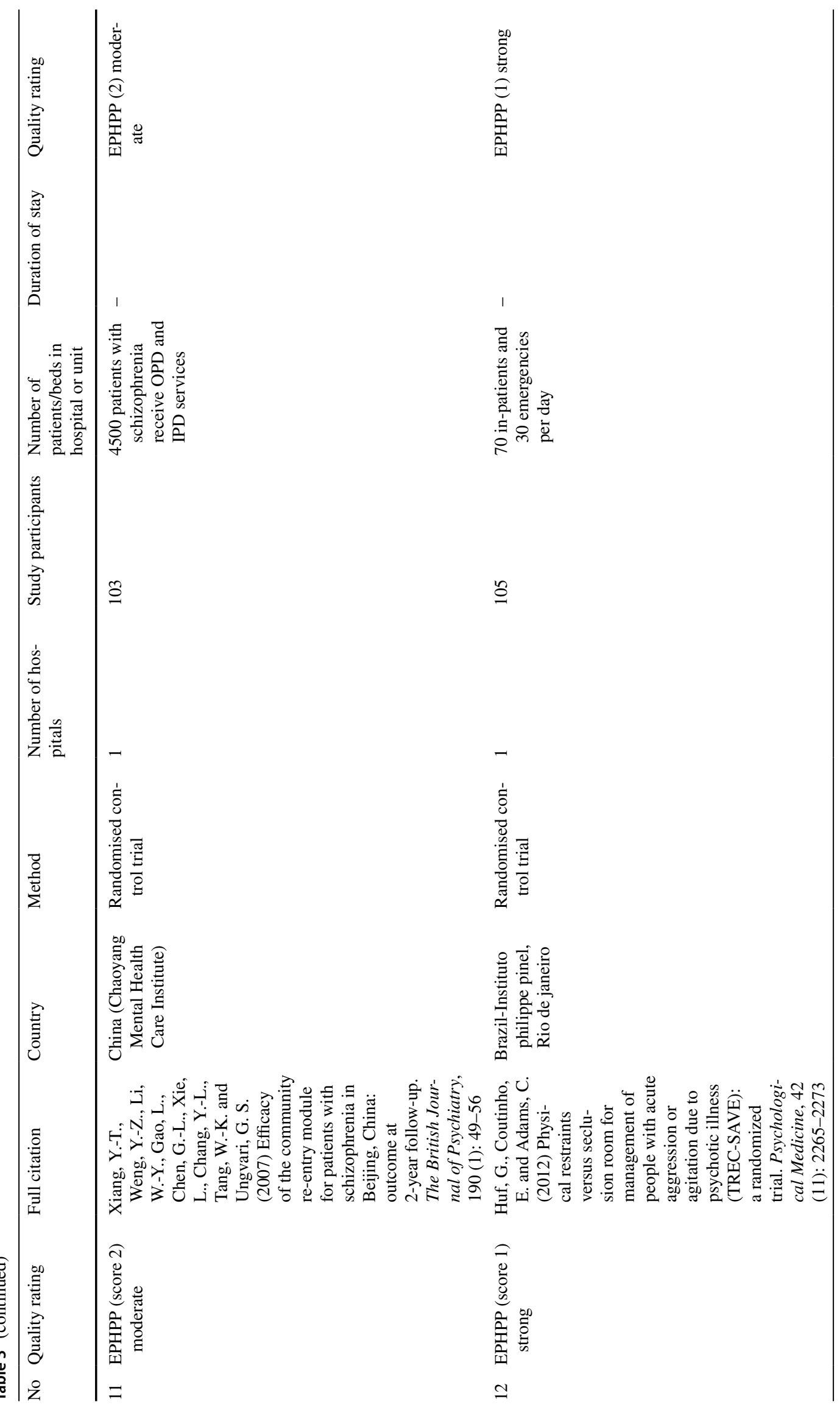




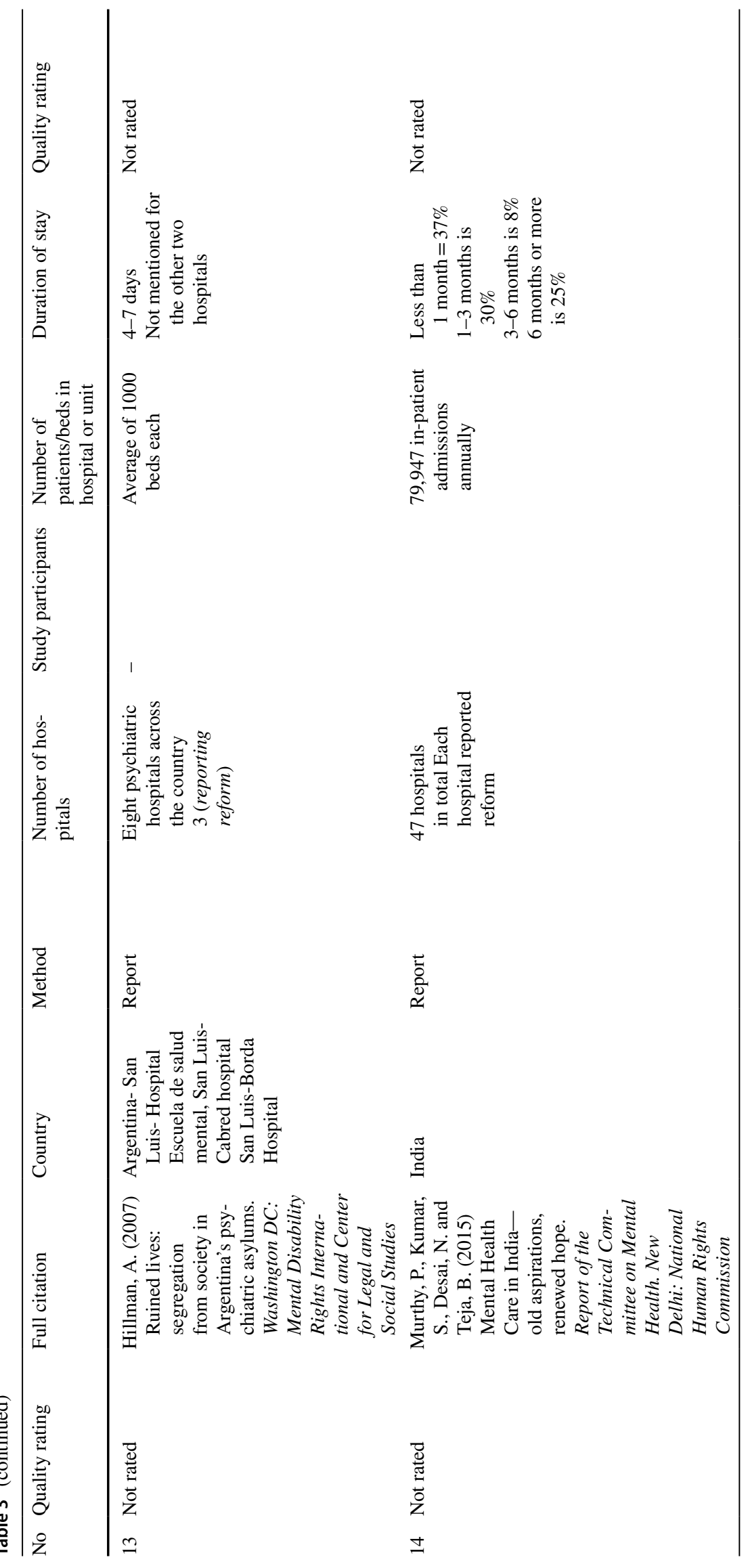




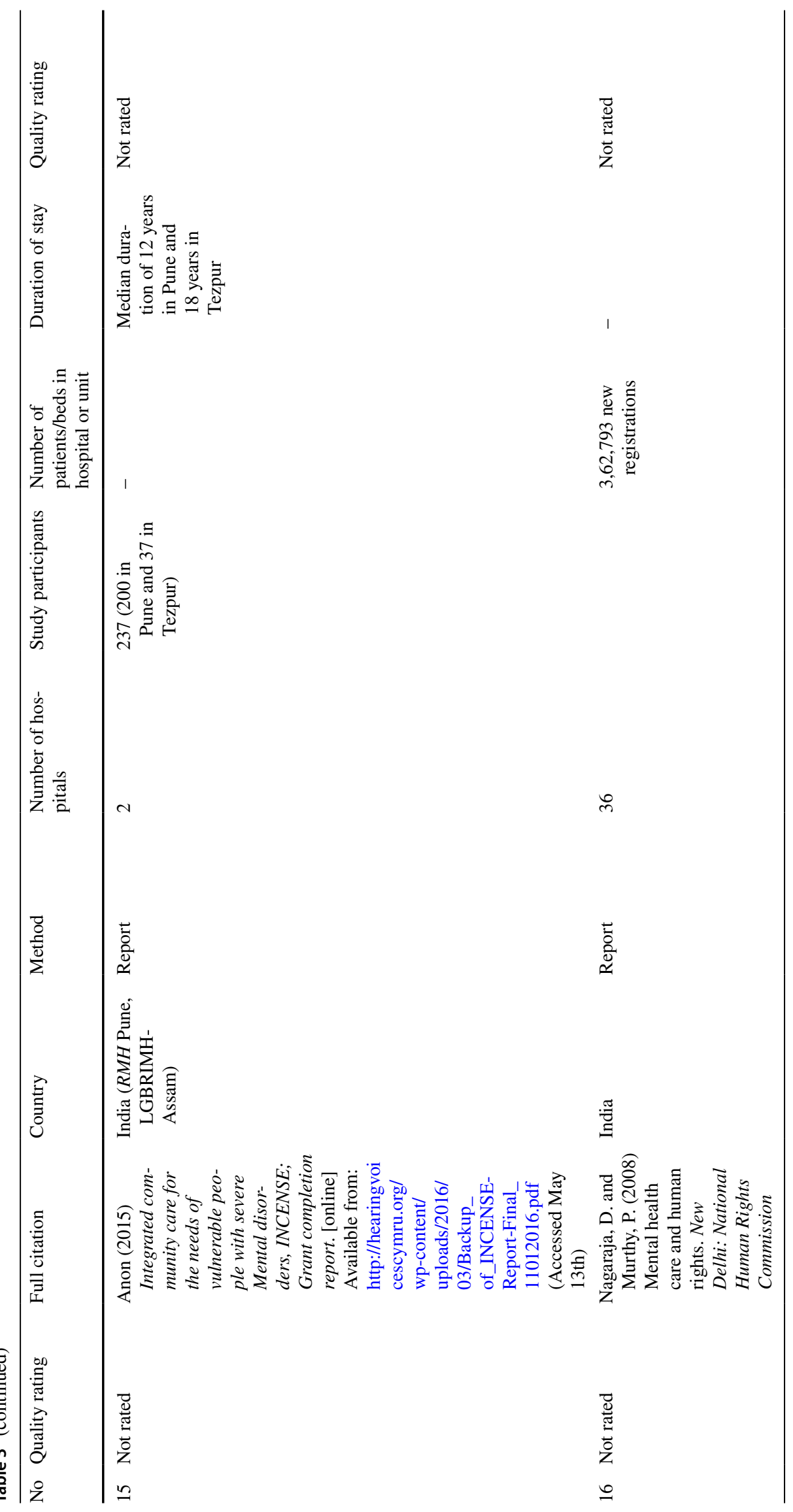


Table 4 Triggers for reform

\begin{tabular}{|c|c|c|c|}
\hline Triggers of reform & No and $\%$ of studies & Country & References \\
\hline Country level transformation of mental health care & $2(12.5 \%)$ & Brazil Grenada & {$[32,37]$} \\
\hline Judicial intervention & $4(25 \%)$ & India, Argentina & {$[19,21,23,31]$} \\
\hline Patients with long duration of hospital stay & $2(12.5 \%)$ & South Africa & {$[27,28]$} \\
\hline Suicide & $1(6.25 \%)$ & China & {$[24]$} \\
\hline Poor quality of life for patients & $3(18.75 \%)$ & China, Sri Lanka & {$[24,34,36]$} \\
\hline Need for evidence-based use of restraint or seclusion & $1(6.25 \%)$ & Brazil & [30] \\
\hline
\end{tabular}

Table 5 Elements of structural reform

\begin{tabular}{|c|c|c|c|}
\hline Elements of structural reform & $\begin{array}{l}\text { Number and } \\
\% \text { of studies }\end{array}$ & Country & References \\
\hline $\begin{array}{l}\text { Closure of a large hospital } \\
\text { and opening of smaller } \\
\text { facilities }\end{array}$ & $1(6.25 \%)$ & Georgia & {$[33]$} \\
\hline Infrastructural improvement & $3(18.75 \%)$ & India & {$[19,21,23]$} \\
\hline $\begin{array}{l}\text { Half way home within the } \\
\text { hospital }\end{array}$ & $1(6.25 \%)$ & Argentina & {$[31]$} \\
\hline Community living services & $2(12.5 \%)$ & India & {$[20,22]$} \\
\hline
\end{tabular}

with discharge planning, structured community re-entry and the involvement of family [26]. Other reforms included introduction of personal/coloured clothes instead of uniforms for patients living in mental hospitals [24].
Argentina shifted towards open wards and reform in medical management of patients in hospital [31] while reforms in Brazil included changes in restraint and seclusion practices [30] and the introduction of mixed gender wards to promote social interaction amongst patients [37]. Process reforms in South Africa composed of changes in medical management and clinical services [28] along with structured discharge planning [27]. Grenada's process reforms composed of changes in medical management and structured discharge planning [32].

Sri Lanka saw a change in restraint and seclusion practices, change in nursing practices (involvement of nursing staff in intake assessment and treatment planning) and changes in the way meals were distributed to patients with the introduction of a buffet-style selfservice system [34].

Table 6 Elements of process reform

\begin{tabular}{|c|c|c|c|}
\hline Elements of process reform & No and $\%$ of studies & Country & References \\
\hline Reform in the process of medical management & $4(25 \%)$ & India, South Africa, Grenada, and Argentina & {$[21,28,31,32]$} \\
\hline $\begin{array}{l}\text { Reform in admission process from custodial to volun- } \\
\text { tary }\end{array}$ & $2(12.5 \%)$ & India & {$[21,23]$} \\
\hline Introduction of open wards & $6(37.5 \%)$ & India, China, and Argentina & {$[21-24,31,36]$} \\
\hline $\begin{array}{l}\text { Introduction of community-based services linked to the } \\
\text { hospital }\end{array}$ & $3(18.75 \%)$ & India, China & {$[22,23,26]$} \\
\hline Reform of restraint and seclusion procedures & $4(25 \%)$ & India, Brazil, Grenada, and Sri Lanka & {$[23,30,32,34]$} \\
\hline $\begin{array}{l}\text { Introduction of mixed gender wards to promote social } \\
\text { interaction }\end{array}$ & $1(6.25 \%)$ & Brazil & [29] \\
\hline Patient involvement in hospital management & $1(6.25 \%)$ & China & {$[24]$} \\
\hline $\begin{array}{l}\text { Promotion of an equal relationship between staff and } \\
\text { patients }\end{array}$ & $1(6.25 \%)$ & China & {$[24]$} \\
\hline Structured discharge planning & $3(18.75 \%)$ & China, Grenada, and South Africa & {$[26,27,32]$} \\
\hline $\begin{array}{l}\text { Change in nursing practice to increase patient interac- } \\
\text { tion }\end{array}$ & $1(6.25 \%)$ & Sri Lanka & {$[34]$} \\
\hline $\begin{array}{l}\text { Introduction of psychosocial interventions including } \\
\text { ADL, employment and other rehabilitation activities }\end{array}$ & $5(31.25 \%)$ & India, China & {$[20,22,24,26,36]$} \\
\hline Engagement with family & $2(12.5 \%)$ & India, China & {$[21,26]$} \\
\hline $\begin{array}{l}\text { Introduction of colored clothes instead of uniforms for } \\
\text { patients }\end{array}$ & $1(6.25 \%)$ & China & {$[24]$} \\
\hline Introduction of a meal management system (buffet) & $1(6.25 \%)$ & Sri Lanka & {$[34]$} \\
\hline
\end{tabular}




\section{Capacity building}

Six (37.5\%) of 16 studies reported capacity building of staff and covered four countries (Table 7). Mental hospitals in India are being reformed as teaching and research institutions with the introduction of formal teaching programs [19, $21,23]$. Grenada reported a formal training programme for staff along with recreational activities, such as a multi-disciplinary journal club, to augment staff capabilities [32]. Mental hospital reforms in Sri Lanka included staff engagement as a means of bringing change in practices [34] and South Africa reported a trained team dedicated to the care of long-stay patients [28].

\section{Outcomes of reform}

Outcome measures were reported by seven (43.75\%) of 16 studies from South Africa, India, China, Brazil and Grenada [22, 24, 26, 27, 30, 32, 36] (Table 8).

In China, clinical outcomes reported improvement in psychiatric symptoms [25, 26], reduction in episodes of relapse [24, 26] along with a reduction in suicides [24]. Functional outcomes reported were an improvement in personal appearance [25] and improvement in engagement with employment $[24,26]$. Social outcomes of reform reported were improvement in staff and patient interactions which was also reported from South Africa
[24, 27], improvement in interaction with family [24] and improvement in overall social functioning [25, 26]. Brazil reported a reduction of time in restraints through the use of seclusion as a technique instead of mechanical restraints [30] and discharge of patients from the hospital [30]. Grenada saw the discharge of patients from hospital as a clinical outcome of reform emphasising short-term care and rapid return of patients to the community. [32]. India reported integration with family as a social outcome of reform [22].

It was particularly interesting to note that none of the studies report data on costs incurred for reform.

\section{Discussion}

This review was undertaken with an objective to bring together research on psychiatric hospital reform in LMICs to understand the process of reform and patient-related outcomes as a result of reform. The review aimed to identify gaps in current evidence and research with regard to the reform of psychiatric institutions in LMICs.

The conceptual framework used for this narrative review was based on the review question and explored the relationship between the circumstances that propelled change or reform in mental hospitals and the elements of reform and patient outcomes associated with the reform.

Table 7 Hospital staff training and capacity building

\begin{tabular}{llll}
\hline Elements of staff training and capacity building & No and \% of studies & Country & References \\
\hline Development of mental hospitals as teaching and research institutes & $3(18.75 \%)$ & India & Grenada \\
Formal training programme for hospital staff & $1(6.25 \%)$ & Sri Lanka 21, 23] & [32] \\
Staff engagement in changing of practice & $1(6.25 \%)$ & South Africa & [34] [28] \\
Trained and dedicated team for management of long-stay patients & $1(6.25 \%)$ & \\
\hline
\end{tabular}

Table 8 Outcomes of reform

\begin{tabular}{|c|c|c|c|c|}
\hline Type of outcome & Outcomes & No and $\%$ of studies & Country & References \\
\hline \multirow[t]{5}{*}{ Clinical } & Improvement in psychiatric symptoms & $2(12.5 \%)$ & China & {$[26,36]$} \\
\hline & Reduction in relapse of illness & $2(12.5 \%)$ & China & {$[24,26]$} \\
\hline & Reduction in time in restraint/isolation & $1(6.25 \%)$ & Brazil & {$[30]$} \\
\hline & Reduction in suicide & $1(6.25 \%)$ & China & {$[24]$} \\
\hline & Discharge of patients from hospital & $2(12.5 \%)$ & Grenada, Brazil & {$[30,32]$} \\
\hline \multirow[t]{2}{*}{ Functional } & Improvement in personal appearance & $1(6.25 \%)$ & China & [36] \\
\hline & Improvement in engagement with employment & $2(12.5 \%)$ & China & {$[24,26]$} \\
\hline \multirow[t]{3}{*}{ Social } & Improvement in staff and patient interaction & $2(12.5 \%)$ & South Africa, China & {$[24,27]$} \\
\hline & $\begin{array}{l}\text { Improvement in interactions with family/integration } \\
\text { with family }\end{array}$ & $2(12.5 \%)$ & China, India & {$[22,24]$} \\
\hline & Improvement in overall social functioning & $2(12.5 \%)$ & China & {$[26,36]$} \\
\hline
\end{tabular}




\begin{tabular}{|c|c|c|c|c|c|}
\hline 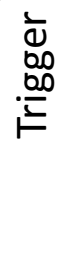 & $\begin{array}{l}\text { What were the } \\
\text { circumstances that } \\
\text { proppelled the reform } \\
\text { process }\end{array}$ & $\begin{array}{l}\frac{E}{2} \\
\frac{0}{0} \\
\frac{1}{\propto}\end{array}$ & $\begin{array}{l}\text { The elements of reform } \\
\text { categorised under } \\
\text { structural reform, process } \\
\text { reform and staff capacity }\end{array}$ & 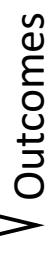 & $\begin{array}{l}\text { Outcome of reform on } \\
\text { individual patients } \\
\text { assessed in terms of } \\
\text { clincal outcomes, } \\
\text { functional outcomes and } \\
\text { social outcomes }\end{array}$ \\
\hline
\end{tabular}

\section{Dearth of research}

There is clearly a dearth of research on mental hospital reform processes. We found only 16 studies from 137 countries across a period of four decades. State-run mental hospitals continue to play a key role in providing services in most parts of the world. They deal with an increasingly challenging population with a large number of people having extended periods of hospital stay [1,3]. Downsizing of mental hospitals and deinstitutionalization comes ridden with its own problems of trans-institutionalization, homelessness and imprisonment of people living with severe mental illness [38-41]. In such a scenario, mental hospitals need to reinvent themselves to meet the needs of the very vulnerable population they serve. Psychiatric hospital reform needs to be backed by robust evidence on the process of reform and its clinical, social and functional outcomes and the costs thereof. This is a key requirement for governments and policy-makers to make informed decisions and improve the landscape of mental health service delivery.

\section{Drivers of reform}

Change or reform appears to be driven by the need to make a difference in the quality of life of long-stay patients [24, 25, $27,28,34]$. Often such reform is catalysed by judicial action or higher-level reform of the country's mental health system as seen in India, Argentina and Brazil [19, 21, 23, 31, 32, 37] The need for improved clinical practice and reduction in violation of basic human rights also triggered reform as evidenced by the modified use of restraints or seclusion as in the case of Brazil [30, 35].

\section{Optimisation of resources}

Mental hospitals in most parts of the world have been established during the colonial era and urgently require refurbishment or renovation of the old infrastructure. As hospitals were downsized, their infrastructure was modified to create facilities that more appropriately serve patient needs.
For instance, using old hospital wards as a half-way-home facility as seen in Argentina [31] and the creation of open wards as in China and Brazil [25, 37]. In India, several infrastructural changes have been carried out across hospitals to improve living conditions for patients [19, 21, 23]. Infrastructural changes have also been associated with the simultaneous development of community living services [20, 22] while downsizing hospitals into more compact acute care units [33].

\section{Process reform as a catalyst to improve quality of life}

Although often unplanned, most reforms seem to be centered around a change in processes. Reform of processeslargely comprising shifts in clinical and medical management protocols is directly linked to improving the quality of life for patients in mental hospitals [21, 23, 26-28, 30-32, $34,35]$. Large-scale shifts, such as changes in admission processes and moving from custodial to voluntary admissions [21, 23], the introduction of open wards [21-25, 31], greater integration of psychosocial services and an incremental push towards improving autonomy and dignity of long-stay patients, have been seen globally [20, 22, 24-26, $34,37]$. Further, greater attention has been paid towards more intangible, and experiential elements of care. Shifts in clothing policies, food service timings and processes, and access to leisure and recreation have all contributed significantly to an improvement in functioning and overall quality of life.

\section{Mental health professionals as key drivers of recovery-oriented practice}

Capacity building of staff was seen as an associated and significant piece of the reform process where countries like India [19, 21, 23] have made a central push for all mental hospitals to transform into centres of excellence that are front runners of training, research and knowledge creation. Training of staff has been, in varying degrees, 
an important conduit of reform in psychiatric hospital settings $[28,32,34]$.

\section{Study limitations}

A major limitation of this review is the variable quality of the studies included with most studies being of poor quality. Further, published literatures from countries that have experienced massive mental health reform, such as that of Brazil [42], are available in languages other than English. Their inclusion was beyond the scope of this review as a result of limited resources available. This also limited our ability to include grey literature in the scope of our review. Detailed quantitative analysis is limited by the quality of included studies as well as the variability in measures. This has implications on the extent of evidence and its ability to answer the question this review focused on which is the extent of scientific evidence around psychiatric hospital reform and its associated patient-related outcomes in the context of lowand middle-income countries.

\section{Conclusion}

Mental hospitals remain an integral part of psychiatric services globally. In some parts of the world, these hospitals form a majority of, and in some cases, the entire service continuum [43]. Mental hospitals however are not static entities, but are evolving and finding renewed relevance in the global landscape of de-institutionalization and communitybased services [2]. Currently, reform of hospitals appears unplanned and de-linked to evidence. Reforms do not appear to be linked to patient outcomes. There is a large gap in scientific evidence that needs to be bridged urgently such that future reform processes may be more informative. Further research could investigate the correlational and causal pathways between reform and patient outcomes, clearly determine the costs of the reforms, and discern whether they require a radical shift in human resource allocations. In addition, we also believe social scientists (psychologists, anthropologists, sociologists, economists, etc.) could study the impact of culture, social norms and value systems on mental health service provision in LMICs.

Acknowledgements Swaran P. Singh and Helena Tuomainen were supported by the National Institute for Health Research (NIHR), Applied Research Centre (ARC), West Midlands. The views expressed are those of the authors and not necessarily those of the NIHR or the Department of Health and Social Care.

Author contributions TR developed the review methodology, extracted the data, and conducted the quality assessment. TR also wrote the first draft of the paper; HT supported the design, protocol development, review and editing of manuscript; KE supported the data extraction, reviewing and editing of the manuscript; SS primary supervisor on the
PhD supported the design, protocol development, review and editing of the manuscript. All authors reviewed the manuscript and accepted the final draft.

Funding This work is supported by Tata Education and Development Trust (TEDT) (DI Regional mental hospital Nagpur, circular number 130) and R G Manudhane Foundation for Excellence (MoU with TEDT dated 10th Aug 2016).

Data availability Not applicable.

\section{Declarations}

Conflict of interest This systematic review is part of the $\mathrm{PhD}$ program undertaken by the PI Tasneem Raja. She is an employee of the Tata Trusts and the Tata Trusts External Individual educational grants program funds the $\mathrm{PhD}$ won on basis of merit.

Ethical approval Not applicable.

Consent to participate Not applicable.

Consent for publication Not applicable.

Code availability. Not applicable.

Open Access This article is licensed under a Creative Commons Attribution 4.0 International License, which permits use, sharing, adaptation, distribution and reproduction in any medium or format, as long as you give appropriate credit to the original author(s) and the source, provide a link to the Creative Commons licence, and indicate if changes were made. The images or other third party material in this article are included in the article's Creative Commons licence, unless indicated otherwise in a credit line to the material. If material is not included in the article's Creative Commons licence and your intended use is not permitted by statutory regulation or exceeds the permitted use, you will need to obtain permission directly from the copyright holder. To view a copy of this licence, visit http://creativecommons.org/licenses/by/4.0/.

\section{References}

1. WHO. Mental health atlas 2017. Geneva: World Health Organization; 2018. World Health Organization WHO MiNDbank. http:// www.who.int/mental_health/mindbank/en. Accessed 1 Sept 2020

2. Chatterjee $S$ (2017) Time to focus on institutional reforms in low and middle income countries. Epidemiol Psychiatr Sci 26(1):1-3. https://doi.org/10.1017/S2045796016000718

3. Fisher WH, Geller JL, Pandiani JA (2009) The changing role of the state psychiatric hospital. Health Aff 28(3):676-684

4. Cohen A, Minas H (2017) Global mental health and psychiatric institutions in the 21st century. Epidemiol Psychiatr Sci 26(1):4-9

5. Dowdall GW (1999) Mental hospitals and deinstitutionalization. Handbook of the sociology of mental health. Springer, pp 519-537

6. Frankish H, Boyce N, Horton R (2018) Mental health for all: a global goal. Lancet 392:1493-1494

7. Patel V, Xiao S, Chen $\mathrm{H}$ et al (2016) The magnitude of and health system responses to the mental health treatment gap in adults in India and China. Lancet 388:3074-3084

8. Collins PY, Patel V, Joestl SS, March D, Insel TR, Daar AS et al (2011) Grand challenges in global mental health. Nature 475(7354):27-30 
9. Chatterjee S, Patel V, Chatterjee A, Weiss HA (2003) Evaluation of a community-based rehabilitation model for chronic schizophrenia in rural India. Br J Psychiatry 182(1):57-62

10. Kleinman A (2009) Global mental health: a failure of humanity. The Lancet 374(9690):603-604

11. Kohn R, Saxena S, Levav I, Saraceno B (2004) The treatment gap in mental health care. Bull World Health Organ 82:858-866

12. Patel V (2007) Mental health in low-and middle-income countries. Br Med Bull 81(1):81-96

13. Saxena S, Thornicroft G, Knapp M, Whiteford H (2007) Resources for mental health: scarcity, inequity, and inefficiency. Lancet 370(9590):878-889

14. Moher D, Altman DG, Liberati A, Tetzlaff J (2011) PRISMA statement. Epidemiology 22(1):128

15. Raja T, Tuomainen H, Madan J, Mistry D, Jain S, Singh S (2020) Psychiatric hospital reform in low-income and middleincome countries structured individualised intervention and recovery (SITAR): a two-arm pragmatic randomised controlled trial study protocol. BMJ Open. https://doi.org/10.1136/bmjop en-2019-035753

16. Godlee F, Jefferson T, Callaham M (2003) Peer review in health sciences. BMJ Books, London

17. Leamy M, Bird V, Le Boutillier C, Williams J, Slade M (2011) Conceptual framework for personal recovery in mental health: systematic review and narrative synthesis. Br J Psychiatry 199(6):445-452

18. Evans N, Lasen M, Tsey K (2015) Appendix A: Effective public health practice project (EPHPP) quality assessment tool for quantitative studies. A systematic review of rural development research briefs in public health. Springer, pp 45-55

19. Cochrane: data collection form for intervention reviews: RCTs and non-RCTs version 3, April 2014. https://dplp.cochrane.org/ data-extraction-forms. Accessed $20 \mathrm{Feb} 2020$

20. Varma S (2016) Disappearing the asylum: modernizing psychiatry and generating manpower in India. Transcult Psychiatry 53(6):783-803. https://doi.org/10.1177/1363461516663437

21. Murthy P, Isaac M, Dabholkar H (2017) Mental hospitals in India in the 21st century: transformation and relevance. Epidemiol Psychiatr Sci 26(1):10-15. https://doi.org/10.1017/S2045796016000755

22. Murthy P, Kumar S, Desai N, Teja B (2016) Mental health care in India-old aspirations, renewed hope. Report of the technical committee on mental health. National Human Rights Commission, New Delhi

23. Integrated community care for the needs of vulnerable people with severe mental disorders (2015) INCENSE; Grant completion report. http://hearingvoicescymru.org/wp-content/uploads/2016/ 03/Backup_of_INCENSE-Report-Final_11012016.pdf. Accessed 13 May 2018

24. Nagaraja D, Murthy P (2008) Mental health care and human rights. National Human Rights Commission, New Delhi

25. Jin D, Li G (1994) The role of human rights and personal dignity in the rehabilitation of chronic psychiatric patients. A rural therapeutic community in Yanbian, Jilin. Br J Psychiatry Suppl 165(24):121-127

26. Fan Z, Huang J, Wu Q, Jiang S (1994) Comparison of standard locked-ward treatment versus open-ward rehabilitation treatment for chronic schizophrenia patients: a one-year controlled trial in Canton. Br J Psychiatry 165(Suppl 24):45-51

27. Xiang Y-T, Weng Y-Z, Li W-Y, Gao L, Chen G-L, Xie L et al (2007) Efficacy of the community re-entry module for patients with schizophrenia in Beijing, China: outcome at 2-year followup. Br J Psychiatry 190(1):49-56
28. Uys LR, Mhlaluka NG, Piper SE (1996) An evaluation of the effect of programme changes in an acute psychiatric unit. Curationis 19(3):21-27

29. Kruger C, Lewis C (2011) Patient and social work factors related to successful placement of long-term psychiatric in-patients from a specialist psychiatric hospital in South Africa. Afr J Psychiatry 14(2):120-129

30. Bandeira PM, Souza CHP, da Silva Guimarães JC, de Almeida Filho AJ, de Almeida Peres MA (2015) Psychiatric nursing in integrated wards accommodating both female and male patients: a historic pioneering reform initiative implemented by the institute of psychiatry, a unit of the federal university of Rio De Janeiro, Brazil. Issues Ment Health Nurs 36(10):791-798

31. Huf G, Coutinho E, Adams C (2012) Physical restraints versus seclusion room for management of people with acute aggression or agitation due to psychotic illness (TREC-SAVE): a randomized trial. Psychol Med 42(11):2265-2273

32. Hillman A (2007) Ruined lives: segregation from society in Argentina's psychiatric asylums. Mental Disability Rights International and Center for Legal and Social Studies, Washington DC

33. Fisher FD, Griffith EE, Mahy GE (1988) Recent developments in the Grenada mental health program. Hosp Community Psychiatry 39(9):980-985

34. Makhashvili N, van Voren R (2013) Balancing community and hospital care: a case study of reforming mental health services in Georgia. PLoS Med 10(1):e1001366. https://doi.org/10.1371/ journal.pmed.1001366

35. Ganesan M (2017) Transforming an out-of-date psychiatric hospital into a patient friendly space: a matter of taking risks. Intervention 15(1):76-81

36. Huf G, Coutinho ES, Ferreira MA, Ferreira S, Mello F, Adams CE et al (2011) TREC-SAVE: a randomised trial comparing mechanical restraints with use of seclusion for aggressive or violent seriously mentally ill people: study protocol for a randomised controlled trial. Trials 12:180. https://doi.org/10.1186/ 1745-6215-12-180

37. Fan Z, Huang J, Wu Q, Jiang S (1994) Comparison of standard locked-ward treatment versus open-ward rehabilitation treatment for chronic schizophrenic patients: a one-year controlled trial in Canton. Br J Psychiatry 165(S24):45-51

38. Bandeira PM, Haddad PSC, da Silva Guimaraes JC, de Almeida Filho AJ, de Almeida Peres MA (2015) Psychiatric nursing in integrated wards accommodating both female and male patients: a historic pioneering reform initiative implemented by the institute of psychiatry, a unit of the federal university of Rio De Janeiro, Brazil. Issues Ment Health Nurs 36(10):791-798. https://doi.org/ $10.3109 / 01612840.2015 .1043674$

39. Lamb HR, Weinberger LE (2001) Persons with severe mental illness in jails and prisons: a review. New Dir Stud Leadersh 2001(90):29-49

40. Thornicroft G, Bebbington P (1989) Deinstitutionalisationfrom hospital closure to service development. Br J Psychiatry 155(6):739-753

41. Carr S (2018) Implementing sustainable global mental health in a fragmenting world. Lancet 392:1497-1498

42. The LP (2015) The land of the free. Lancet Psychiatr 2(5):363

43. Candiago RH, Saraiva Sda S, Goncalves V, Belmonte-de-Abreu P (2011) Shortage and underutilization of psychiatric beds in southern Brazil: independent data of Brazilian mental health reform. Soc Psychiatry Psychiatr Epidemiol 46(5):425-429. https://doi. org/10.1007/s00127-010-0207-1 